\title{
BMJ Open Early Diagnosis of Gestational Diabetes Mellitus (EDoGDM) study: a protocol for a prospective, longitudinal cohort study
}

\author{
Bin Liu, ${ }^{1}$ Yun $\mathrm{Xu},{ }^{2}$ Ying Zhang, ${ }^{1}$ Jian Cai, ${ }^{1}$ Langhui Deng, ${ }^{3}$ Jianbo Yang, ${ }^{1}$ Yi Zhou, ${ }^{1}$ \\ Yuhang Long, ${ }^{1}$ Jinxin Zhang, ${ }^{4}$ Zilian Wang ${ }^{1}$
}

To cite: Liu B, Xu Y, Zhang $Y$, et al. Early Diagnosis of Gestational Diabetes Mellitus (EDoGDM) study: a protocol for a prospective, longitudinal cohort study. BMJ Open 2016;6:e012315. doi:10.1136/bmjopen-2016012315

- Prepublication history for this paper is available online. To view these files please visit the journal online (http://dx.doi.org/10.1136/ bmjopen-2016-012315).

Received 18 April 2016 Revised 7 October 2016 Accepted 13 October 2016

CrossMark

For numbered affiliations see end of article.

Correspondence to Dr Zilian Wang;

wzlsums@163.com

\section{ABSTRACT}

Introduction: A diagnosis of gestational diabetes mellitus (GDM) in low-risk pregnant women is based on an oral glucose tolerance test (OGTT) between 24 and 28 gestational weeks. However, there is insufficient evidence for why the test is performed in this time period. Moreover, the fetus may be exposed to hyperglycaemia prior to the current testing time frame, making earlier administration potentially advantageous. The main purpose of the present study is to investigate the GDM diagnostic value of an OGTT performed at 18-20 gestational weeks. The results of the study may provide scientific insight into the most beneficial time of OGTT for pregnant women.

Methods and analysis: As a prospective, longitudinal cohort study, the Early Diagnosis of Gestational Diabetes Mellitus (EDoGDM) study will recruit 570 pregnant women who meet the inclusion and exclusion criteria outlined below. OGTTs will be performed between 18 and 20 gestational weeks (early OGTT) and 24-28 gestational weeks (regular OGTT). Clinical and laboratory information of the mother and their offspring will be collected for analysis. The prevalence of GDM at 18-20 gestational weeks will be described, and the sensitivity, specificity, positive predictive value and negative predictive value of early OGTT on diagnosis of GDM will be studied. Clinical outcomes associated with hyperglycaemia will be compared between groups diagnosed by early or regular OGTT.

Ethics and dissemination: The study was approved by The Ethical Committees of The First Affiliated Hospital of Sun Yat-sen University (number 2016-042). Signed informed consent will be obtained from all participants. The results of this study will be disseminated in peer-reviewed journals.

Trial registration number: NCT02740283.

\section{INTRODUCTION}

Gestational diabetes mellitus (GDM) is a condition of glucose intolerance that begins or is first recognised during pregnancy. ${ }^{1}{ }^{2}$

\section{Strengths and limitations of this study}

- The Early Diagnosis of Gestational Diabetes Mellitus (EDoGDM) study involves prospective follow-up during pregnancy to ascertain longitudinal metabolic status in pregnant women and its relationship to pregnancy outcomes.

- The EDoGDM study will provide scientific evidence on the significance of an oral glucose tolerance test (OGTT) earlier than the classically tested time period for diagnosis of gestational diabetes mellitus (GDM). The results may improve the clinical outcomes of mothers complicated with GDM and their offspring by early diagnosis and management of hyperglycaemia.

- In the event that sample size in a subgroup is small, a direct association between hyperglycaemia in early OGTT and adverse clinical outcomes may be difficult to analyse. The representative nature of the sample is a potential limitation, as it is subject to possible selection bias.

Currently, most guidelines recommend an oral glucose tolerance test (OGTT) between 24 and 28 gestational weeks as the method of diagnosis for GDM. ${ }^{1}{ }^{3-7}$ According to the definition, GDM can develop at any time during pregnancy; however, most GDM cases are diagnosed after 24 weeks.

Following diagnosis, proper management (glycaemic monitoring, lifestyle changes, nutritional counselling, exercise and insulin use if appropriate, etc) of GDM in pregnant women will be beneficial in controlling maternal and neonatal short-term complications. ${ }^{2}$ However, offspring of mothers with GDM are still at a higher risk for developing diabetes, ${ }^{8}$ obesity $^{8-10}$ and metabolic disorders ${ }^{11}$ in the long term. One potential reason is that these offspring were exposed to maternal hyperglycaemia prior to diagnosis. Therefore, current guidelines have noticed that early diagnosis 
of GDM may improve maternal, fetal and neonatal outcomes. ${ }^{361213}$ However, there is limited evidence on the benefits of diagnosing and treating GDM before 24-28 gestational weeks. ${ }^{3} 61213$

The evidence of the regular time period (24-28 gestational weeks) for OGTT test in low-risk pregnant women has not been clarified. In 1964, the OGTT was first set up as diagnostic criteria of GDM by O'Sullivan and Mahan $;{ }^{14}$ however, the gestational week for examination was not defined. The National Diabetes Data Group $(\mathrm{NDDG})^{15}$ and the Third International WorkshopConference on Gestational Diabetes Mellitus ${ }^{16}$ adopted O'Sullivan and Mahan's method with some modification in 1979 and 1991, but still a time point for the OGTT was not suggested (table 1).

To the best of our knowledge, the first recommendation to perform an OGTT in low-risk pregnant women at 24-28 gestational weeks was raised by the French College of Gynecologists and Obstetricians (CNGOF) in 1996. ${ }^{17}$ The European Association for the Study of Diabetes (EASD) also suggested a one-step OGTT at 28 gestational weeks in the same year. ${ }^{18}$ In 2000, the American Diabetes Association (ADA) began to recommend an OGTT at 24-28 gestational weeks to screen for GDM in pregnant women using the Carpenter criteria (the diagnostic cut-off was set at 5.3-10.0-8.6 mmol/L in $75 \mathrm{~g}$ OGTT) ${ }^{19}$ Although most of these guidelines recommend an OGTT at 24-28 gestational weeks, none provided robust evidence for the reason behind this time point.

For more than two decades, the OGTT has helped to identify pregnant women who are at risk for developing diabetes after pregnancy, ${ }^{14}$ but this criteria was not designed to identify risk of adverse perinatal outcomes. ${ }^{20}$ To investigate the adverse perinatal outcomes associated with the result of the OGTT, the Hyperglycemia and
Adverse Pregnancy Outcomes (HAPO) study was set up. The study recruited 25505 pregnant women and found an association between mild hyperglycaemia in the OGTT with increased birth weight and increased cord blood C peptide. ${ }^{20}$

According to the result of the HAPO study, the International Association of Diabetes and Pregnancy Study Groups (IADPSG) reset the diagnostic cut-off of the OGTT to $5.1-10.0-8.5 \mathrm{mmol} / \mathrm{L}$ in $2010^{6}$. Further, the $\mathrm{ADA},{ }^{21} \mathrm{WHO}^{12}$ and the International Federation of Gynecology and Obstetrics (FIGO) ${ }^{13}$ also adjusted their criteria for screening GDM in 2011, 2013 and 2015, respectively. The result of the HAPO study is drawn from the OGTT at 24-32 gestational weeks; however, the guidelines still recommend 24-28 gestational weeks as the time point for the OGTT.

The reasons why we should investigate the most appropriate time for the OGTT in pregnant women are as follows: initially, the time point for the OGTT was not set (from O'Sullivan and Mahan's study in 1964 to the Third International Workshop-Conference on Gestational Diabetes Mellitus in 1991). Second, Carpenter and Coustan ${ }^{22}$ suggested performing the OGTT after 24 gestational weeks since most patients demonstrated insulin resistance at this time; however, insulin resistance is known to begin during the second trimester. ${ }^{23}$ Third, after the HAPO study, the OGTT was used to identify adverse neonatal outcomes; however the impact of hyperglycaemia on the fetus may exist before an OGTT between 24 and 28 weeks.

The main purpose of our study is to evaluate an OGTT at 18-20 gestational weeks as an early diagnosis method of GDM in low-risk pregnant women. By investigating the consistency of early and regular OGTT results, as well as maternal-neonatal outcomes that are

\begin{tabular}{|c|c|c|c|}
\hline Year & Author or organisation & Gestational age & Main contribution or changes \\
\hline 1964 & O'Sullivan and Mahan & No recommendation & Set up OGTT as diagnostic criteria of GDM \\
\hline 1979 & NDDG & No recommendation & Test plasma rather than whole blood to increase reproducibility \\
\hline 1982 & Carpenter and Coustan & 24-33 & $\begin{array}{l}\text { Apply the glucose oxidase method rather than the Somogyi-Nelson } \\
\text { method to avoid the measurement of other substances }\end{array}$ \\
\hline 1991 & The 3rd Workshop & No recommendation & Apply $75 \mathrm{~g}$ glucose rather than $100 \mathrm{~g}$ glucose \\
\hline 1996 & CNGOF & $24-28$ & First recommend gestational age \\
\hline 1996 & EASD & 28 & \\
\hline 2000 & ADA & 24-28 & Test fasting, 1 and 2 hours \\
\hline 2008 & HAPO study & 24-32 & Focus on perinatal outcome associated with OGTT value \\
\hline 2010 & IADPSG & $24-28$ & Adjust diagnosis cut-off \\
\hline 2011 & ADA & 24-28 & \\
\hline 2013 & WHO & $24-28^{*}$ & \\
\hline 2015 & ADA & $24-28$ & \\
\hline 2015 & FIGO & 24-28 & \\
\hline
\end{tabular}

${ }^{*}$ The WHO-2013 guideline recommended that an OGTT could be performed at any time during pregnancy for diagnosis of GDM, but as an update of the WHO-1999 version, the formal systematic testing for GDM is between 24 and 28 gestational weeks. ADA, American Diabetes Association; CNGOF, French College of Gynecologists and Obstetricians; EASD, European Association for the Study of Diabetes; FIGO, International Federation of Gynecology and Obstetrics; GDM, gestational diabetes mellitus; HAPO, Hyperglycemia and Adverse Pregnancy Outcomes; IADPSG, International Association of Diabetes and Pregnancy Study Groups; NDDG, National Diabetes Data Group; OGTT, oral glucose tolerance test; The 3rd Workshop, The 3rd International Workshop-Conference on GDM. 
associated with the early OGTT results, this study will provide a scientific basis for the most appropriate time to perform an OGTT in pregnant women.

\section{METHODS AND ANALYSIS}

Study design and settings

The Early Diagnosis of Gestational Diabetes Mellitus (EDoGDM) study is a prospective, longitudinal cohort study. The study will be carried out in the First Affiliated Hospital of Sun Yat-sen University (SYSU), Guangzhou, China. As the national key clinical specialist in South China, the department of obstetrics is providing prenatal care for about 5000 pregnant women every year, and about 4000 pregnant women deliver in our centre in a year.

\section{Study population}

Inclusion criteria

Singleton pregnant women will be invited to participate in this study if they are between 18 and 40 years of age, and have had their first prenatal visit in our centre before 18 weeks 0 days of gestation.

\section{Exclusion criteria}

There is a clear recommendation to screen for GDM in high-risk pregnant women before 24 and 28 gestational weeks in many guidelines, ${ }^{3} 57$ 24-26 and thus women will be excluded from the study if they have any of the following risk factors or conditions:

1. History of GDM or pre-existing diabetes mellitus (DM)

2. Family history of DM (first-degree relative with diabetes or a sister with GDM)

3. Body mass index $(\mathrm{BMI})>30 \mathrm{~kg} / \mathrm{m}^{2}$

4. Previous macrosomia (baby with birth weight $>4000 \mathrm{~g}$ ) or a history of stillbirth

5. Polycystic ovary syndrome

6. Medications: corticosteroids, antipsychotics

7. Participant not willing to take OGTT twice (at 18-20 gestational weeks and 24-28 gestational weeks), or not willing to have a series of prenatal care visits and deliver in our centre.

The exclusion criteria are according to the guidelines of IADPSG (2015 version) with some modifications: first, previously elevated blood glucose levels were not applied because no cut-off or frequency was provided in the guideline. Second, pregravid obesity was considered high risk, so individuals with maternal pregravid BMI larger than $30 \mathrm{~kg} / \mathrm{m}^{2}$, rather than $35 \mathrm{~kg} / \mathrm{m}^{2}$, were excluded. Third, macrosomia was defined as birth weight $>4000 \mathrm{~g}$, rather than $4500 \mathrm{~g}$ according to Chinese guidelines.

All the women who participate in the study will provide written informed consent.

\section{Sample size}

The main purpose of our study is to evaluate an OGTT at 18-20 gestational weeks as a potential earlier diagnostic screen for GDM in low-risk pregnant women; therefore, the two OGTT results (early OGTT at 18-20 gestational week and regular OGTT at 24-28 gestational week) would be compared. For this purpose, the sample size estimation method for diagnostic study was applied, ${ }^{27}$ and the following assumptions were made: a confidence level of 0.95 , a precision of estimate (maximum marginal error) of $10 \%$ in a bilateral contrast, for a sensitivity of $80 \%$ and specificity of $70 \%$ in the early OGTT and prevalence of $12 \%$ in the research population. The overall sample size required 512 and 91 participants based on sensitivity and specificity, respectively. Given these assumptions, and estimating that $\sim 10 \%$ of individuals would miss follow-up appointments or be diagnosed as overt DM complicated with pregnancy, the final sample size was adjusted to 570 participants.

\section{Participant selection and recruitment}

The process of participant selection and recruitment will be divided into three stages (figure 1). First, potential participants that meet the inclusion criteria will be invited by a research assistant and the objective, procedure, benefits and risks of the study will be explained.

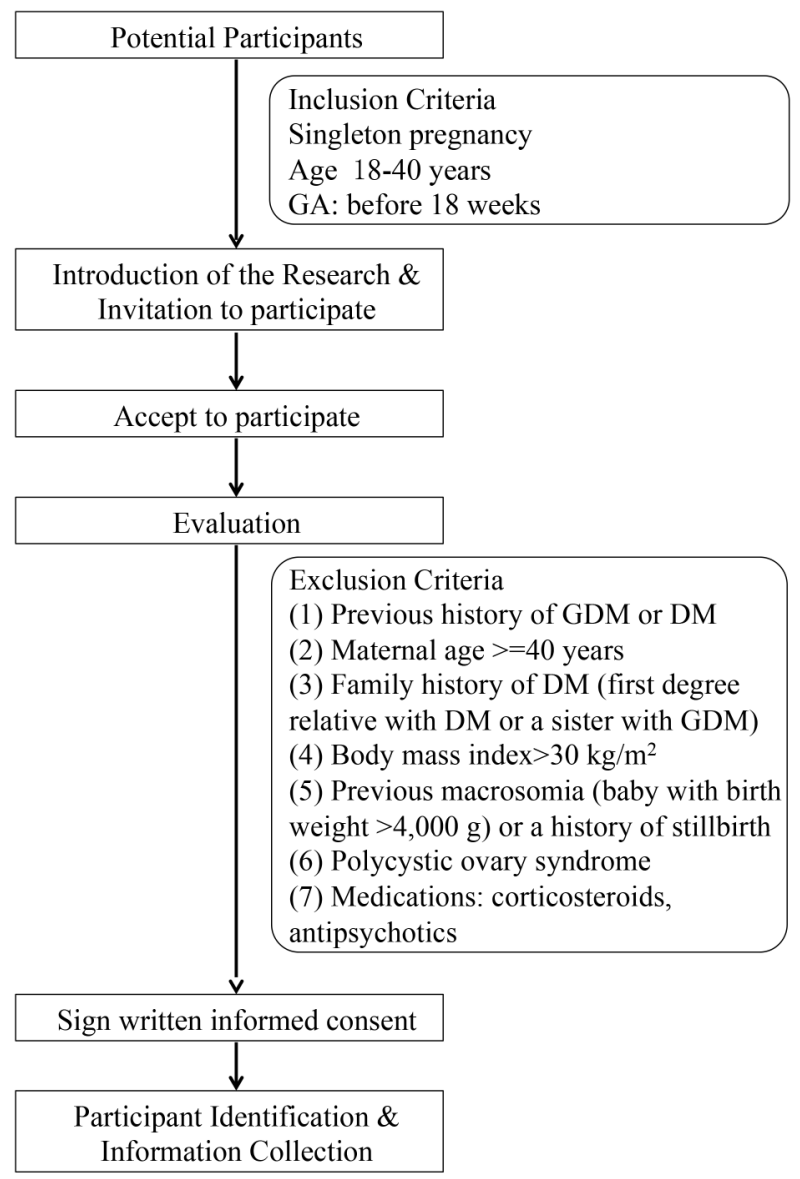

Figure 1 Process of participant selection and recruitment of the EDoGDM study. DM, diabetes mellitus; EDoGDM, Early Diagnosis of Gestational Diabetes Mellitus; GA, gestational age; GDM, gestational diabetes mellitus. 
Second, participants who are interested in participating will be evaluated by researchers and research assistants for exclusion criteria. Third, participants who are finally selected will provide written informed consent prior to the start of the study.

Once selected, each participant will be given an identification number. This ID will be linked to other information unique to the participant, including name, age, citizen ID, outpatient ID and hospital ID. In order to minimise the loss of follow-up, up to two telephone numbers for the participant and her relative will be recorded.

\section{Procedures}

The follow-up for each participant in this study will begin at the first prenatal visit and terminate 42 days postpartum. Follow-up will be performed in a prenatal care clinic and inpatient section of the department of obstetrics. Participants will be assessed at eight time points as described below and summarised in figure 2, and details of the assessment are shown in table 2:

1. Initial assessment (before 18 gestational weeks)

Participants will be recruited before 18 weeks 0 days of gestation. The initial assessment will be performed immediately following participant recruitment. The participant's ethnicity, employment status, medical history, gravity, parity, maternal height and maternal weight before pregnancy will be recorded. Maternal weight, blood pressure and fasting plasma glucose (FPG) will be measured at the time of recruitment.

The confirmation of gestational age was in accordance with the method used in the HAPO study. ${ }^{20}$ Briefly, if the date of the last menstrual period (LMP) is certain, gestational age and expected date of delivery are able to
Figure 2 Study flow chart. From the first prenatal visit to 42 days postpartum, the participants will be assessed at eight time points. The study will begin before 18 gestational weeks with an initial assessment. Early and regular OGTT tests will be performed at 18-20 and 24-28 gestational weeks, respectively. Subsequent to the regular OGTT test, participants will be divided into four groups: (1) early GDM, pregnant women who were diagnosed as GDM both in the early and regular OGTT; (2) late GDM, pregnant women whose OGTT value is normal in the early OGTT but diagnosed as GDM in the regular OGTT; (3) potential GDM, pregnant women diagnosed as GDM in the early OGTT but do not meet the criteria in the regular OGTT; (4) NGT, pregnant women have normal values in both OGTTs.

Management of GDM will be provided to the early and late GDM groups and information of prenatal visit will be collected in 30-32, 33-36 and after 37 gestational weeks. After delivery, pregnancy outcomes will be compared between the early and late GDM groups, and between the potential GDM and NGT groups. DM, diabetes mellitus; GDM, gestational diabetes mellitus; GW, gestational week; NGT, normal glucose tolerance; OGTT, oral glucose tolerance test.

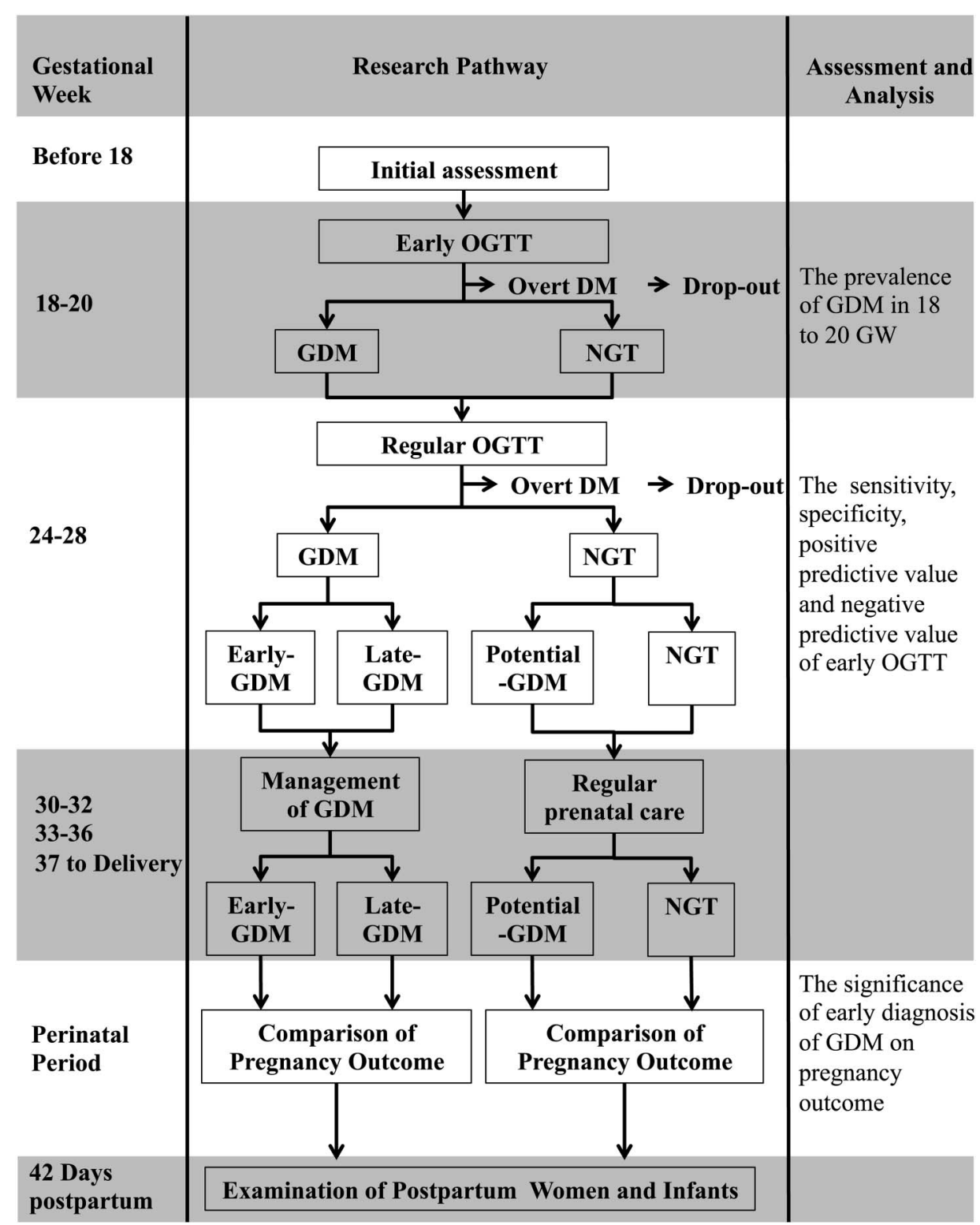


Table 2 Summary of information collected at every stage

\begin{tabular}{|c|c|c|c|c|c|c|c|c|}
\hline \multirow{2}{*}{$\begin{array}{l}\text { Event } \\
\text { Gestational week }\end{array}$} & \multirow{2}{*}{$\begin{array}{l}\begin{array}{l}\text { Initial } \\
\text { assessment }\end{array} \\
\text { Before } 18\end{array}$} & \multirow{2}{*}{$\begin{array}{l}\text { Early } \\
\text { OGTT } \\
18-20\end{array}$} & \multirow{2}{*}{$\begin{array}{l}\text { Regular } \\
\text { OGTT } \\
24-28\end{array}$} & \multicolumn{3}{|c|}{ Prenatal visits } & \multirow{2}{*}{$\begin{array}{l}\text { Perinatal } \\
\text { period } \\
\text { Time of } \\
\text { delivery }\end{array}$} & \multirow{2}{*}{$\begin{array}{l}\text { Post partum } \\
42 \text { days post } \\
\text { partum }\end{array}$} \\
\hline & & & & $30-32$ & $33-36$ & $\begin{array}{l}37 \text { to } \\
\text { delivery }\end{array}$ & & \\
\hline \multicolumn{9}{|l|}{ Clinical information } \\
\hline Maternal BMI & $x$ & $x$ & $x$ & $x$ & $x$ & $x$ & $x$ & $x$ \\
\hline Blood pressure & $\times$ & $x$ & $\times$ & $x$ & $\times$ & $x$ & $x$ & $x$ \\
\hline Fundal height & $\times$ & $\times$ & $\times$ & $\times$ & $\times$ & $\times$ & $\times$ & \\
\hline Abdominal circumference & $x$ & $x$ & $x$ & $x$ & $x$ & $x$ & $x$ & $x$ \\
\hline Medical complications & $x$ & $x$ & $x$ & $x$ & $x$ & $\times$ & $\times$ & $x$ \\
\hline \multicolumn{9}{|l|}{ Laboratory information } \\
\hline OGTT & & $\times$ & $\times$ & & & & & * \\
\hline Fasting glucose & $x$ & & & $x$ & $x$ & $x$ & $x$ & $x$ \\
\hline Fasting insulin & & $x$ & $x$ & & & & $x$ & \\
\hline Fasting C peptide & & $\times$ & $\times$ & & & & $\times$ & \\
\hline Fasting lipid profile & & $\times$ & $\times$ & & & & $x$ & \\
\hline HbA1c & & $x$ & $x$ & & & & $x$ & \\
\hline
\end{tabular}

be estimated. Ultrasonography will be performed between 6 and 24 weeks of gestation to confirm gestational age. If the estimated gestational age based on the LMP differs from that based on ultrasonography (more than 5 days between 6 and 13 gestational weeks, or more than 10 days between 14 and 24 gestational weeks), the gestational age and expected date of delivery will be adjusted according to the ultrasonography result. If the LMP is uncertain, gestational age will be determined by the ultrasonography result alone.

2. Early OGTT (18-20 gestational weeks)

In this step, and the following prenatal visits, participants will visit the prenatal care clinic and maternal weight, blood pressure, fundal height, abdominal circumference and medical complications will be recorded.

Early OGTT will be performed between 18 and 20 gestational weeks. After an overnight fast, pregnant women will complete a 2 hour $75 \mathrm{~g}$ OGTT. Samples will be analysed by the Department of Laboratory Medicine of the First Affiliated Hospital of SYSU. Fasting plasma insulin, C peptide, lipid profile and haemoglobin A1c (HbA1c) levels will also be examined.

Results of the early OGTTs will be kept confidential by the research assistants. Participants and clinicians will not be aware of the early OGTT results until the study is completed. Participants whose plasma glucose levels are $\geq 7.0 \mathrm{mmol} / \mathrm{L}$ (fasting) or $11.1 \mathrm{mmol} / \mathrm{L}$ ( 2 hours postprandial) by OGTT will be considered overt DM complicated with pregnancy. This result will be disclosed to both the participant and clinician and these cases will be excluded from this study; however, our centre will continue the management and therapy of these patients.

Participants whose plasma glucose value by OGTT is $\geq 5.1 \mathrm{mmol} / \mathrm{L}$ (fasting), or $10.0 \mathrm{mmol} / \mathrm{L}$ (1 hour) or $8.5 \mathrm{mmol} / \mathrm{L}$ (2 hours) will be considered as potential patients with GDM. Currently, there is not enough evidence to diagnose GDM in low-risk pregnant women by OGTT in this time period; ${ }^{5} 1224$ therefore, the early GDM group will still follow up without therapy.

3. Regular OGTT (24-28 gestational weeks)

The procedure of OGTT and information collection will be the same as the early OGTT step. Therapy and glucose monitoring will be provided to the participants who meet the criteria of GDM or overt DM complicated with pregnancy, but the study of the latter will be terminated.

At this stage, participants will be divided into four subgroups (figure 2): pregnant women who were diagnosed as GDM both in the early and regular OGTT will be in the early GDM group; whose OGTT value is normal in the early OGTT but diagnosed as GDM in the regular OGTT step will be in the late GDM group; those diagnosed as GDM in the early OGTT but do not meet the criteria in the regular OGTT step will be in the potential GDM group; and those who have normal values in both OGTTs will be in the normal glucose tolerance (NGT) group. According to the therapeutic strategy, both the early GDM and late GDM groups will be treated.

Therapy of GDM will be performed in our centre by experts in obstetrics, metabolomics and nutrition, in an outpatient clinic or in hospital, according to clinical guidelines. Briefly, there are four important therapeutic domains.

First, each pregnant woman with GDM will be monitored for glycaemia, weight gain and fetal well-being during pregnancy. Second, lifestyle modification will be recommended for each patient with GDM, including nutrition education and guidance of physical activity. Third, if hyperglycaemia continues or becomes worse following lifestyle modification, insulin therapy will be provided subsequent to discussion with an 
endocrinologist. Oral antidiabetic agents are not considered in this study, because there is no long-term evidence for the safety of these agents and nor are these agents recommended for GDM management in Chinese guidelines. Fourth, suggestion of timing and mode of delivery will be provided by a consultant obstetrician when the patient comes to term.

4-6. Prenatal visits (30-32 gestational weeks, 33-36 gestational weeks, and 37 gestational weeks to delivery)

In these stages, pregnant women will receive prenatal care in the indicated period. Maternal weight, blood pressure, fundal height, abdominal circumference and medical complications will be recorded and capillary blood glucose will be tested in a fasting state.

If the participants receive additional prenatal care in a period because of medical complications, the history will be recorded. However, for consistency within the cohort, only the information for one prenatal visit in a period will be analysed.

\section{Perinatal period}

At this stage, participants will be hospitalised and evaluated in the department of obstetrics. Maternal weight, blood pressure, gestational age of delivery, perinatal complications, route of delivery and progression of labour will be recorded. FPG, insulin, C peptide, HbAlc and lipid profiles will be examined.

For the neonates, birth weight, length, head, shoulder and abdominal circumferences, Apgar score and neonatal complications will be recorded. Umbilical cord plasma glucose, insulin, $\mathrm{C}$ peptide, HbAlc and lipid profiles will be examined.

\section{Postpartum (42 days after delivery)}

At this stage, participants will be invited to the postpartum clinic and maternal weight and blood pressure will be recorded. FPG, insulin, C peptide and lipid profiles will be examined. For those complicated with GDM (in the early, late and potential GDM groups), an OGTT will be performed to screen for postpartum DM.

For the infants, weight, length, head, shoulder and abdominal circumferences, and any complications will be recorded.

\section{STUDY OUTCOME AND DATA ANALYSIS}

To investigate whether an early OGTT could be used to diagnose GDM at 18-20 gestational weeks, the primary focus of this study is to compare the results of early OGTT and regular OGTT (at 24-28 gestational weeks) on diagnosis of GDM. According to the research procedure, we can divide the study into three stages, and therefore three outcomes will be analysed (figure 2).

In the first stage, after all participants finish the early OGTT, the prevalence of GDM at 18-20 gestational weeks will be clearer. In the second stage, after all participants complete the regular OGTT, the results of early and regular OGTTs will be compared and the sensitivity, specificity, positive predictive value and negative predictive value of early OGTT on diagnosis of GDM will be studied. The third stage analysis will be performed after all participants complete the study. To investigate the significance of early diagnosis of GDM, we will analyse the clinical outcome between early GDM and late GDM groups, both of which received therapy, and we will also compare the clinical outcomes between the potential GDM and NGT groups, which did not receive therapy.

\section{ETHICS AND DISSEMINATION}

The study has little to no risk to participants. Signed informed consent will be obtained from all participants. Participation in the study will not interfere with the regular prenatal care or management of GDM in pregnant women.

The results of this study will be disseminated in peerreviewed journals.

\section{DISCUSSION}

GDM now affects about $17.5 \%$ of pregnant women in the Chinese population, ${ }^{28}$ and the prevalence is also increasing in the global population. ${ }^{12}$

Early diagnosis of GDM would play an important role in the prevention of short-term and long-term complications that affect both the mothers and their offspring. However, in current clinical practice, the diagnosis of GDM is mainly based on an OGTT between 24 and 28 gestational weeks and therapy typically begins in the third trimester, which may not be early enough for prevention of complications.

Different strategies have been studied for early diagnosis of GDM. We joined a multicentre investigation on the predictive value of FPG for subsequent GDM. The result of the study showed that about two-thirds of pregnant women whose $\mathrm{FPG} \geq 6.10 \mathrm{mmol} / \mathrm{L}$ at the first prenatal visit would develop GDM, but an OGTT was still recommended at 24-28 gestational weeks. ${ }^{28}$ Following this study, we found that the FPG level at the first prenatal visit was associated with neonatal birth weight. ${ }^{29}$ Recently, we reported that FPG at the first prenatal visit was associated with neonatal birth head circumference and shoulder circumference, but no association was found between FPG and mid-gestational lipid profiles. ${ }^{30}$ These studies demonstrate that although FPG is associated with maternal metabolic disorders and neonatal birth size, a single FPG test is not able to diagnose GDM.

The OGTT is a well-recognised method for the diagnosis of GDM that has been used for more than 50 years. Compared with the original method of O'Sullivan and Mahan, ${ }^{14}$ many details have been well investigated and changed to improve the quality of the test (table 1), including the use of plasma to replace whole blood, ${ }^{15}$ the application of the oxidase method to avoid sample contamination by other substances, ${ }^{22}$ a reduction in the amount of glucose required, ${ }^{16}$ and the application of 2 hour results to replace 3 hour test results. ${ }^{19}$ However, the reason for the gestational time point of examination was not clarified. 
Recently, a substantial alteration of OGTT in many guidelines ${ }^{13-5} 713$ is the reduction of the cut-off value, following the HAPO study. This alteration is due to the consideration of adverse perinatal outcomes that are associated with hyperglycaemia. ${ }^{20}$ However, the suggested time point of OGTT in the guidelines has not been moved forward. Considering that hyperglycaemia may exist before 24-28 gestational weeks, and have an adverse impact on the developing fetus, we designed this study to investigate the diagnostic value of an OGTT earlier than the classically tested time period.

In the present protocol, the early OGTT will be performed between 18 and 20 gestational weeks. This selected time period is based on three factors. First, insulin resistance begins during the second trimester, ${ }^{31}$ so the time period should be chosen from 13 to 24 gestational weeks. Second, our recent study in a Chinese population showed that pregnant women with GDM had similar FPG between 16-20 and 20-24 gestational weeks. ${ }^{29}$ Moreover, a previous study found that only about one-third of mothers with GDM could be diagnosed by OGTT before 16 gestational weeks. ${ }^{32}$ Therefore, an examination period of the early OGTT was chosen of 16-20 gestational weeks. Third, the reproducibility of OGTT is increased at later gestational ages; ${ }^{17}$ thus, we chose $18-20$ gestational weeks as the time period of the early OGTT, since it may provide 4-10 additional weeks for the management of GDM with considerable true-positive rate.

In many current guidelines, there is a clear recommendation to perform an OGTT in high-risk women during the first trimester, or as early as the first prenatal visit; ${ }^{3} 57$ 24-26 therefore, pregnant women with one or more risk factors will be excluded in this study. Guidelines also revealed that there is not enough evidence to screen and treat GDM in low-risk women before 24 gestational weeks; ${ }^{5} 12{ }^{24}$ thus, therapy for GDM will be based on the regular OGTT result between 24 and 28 gestational weeks.

A potential limitation in this study is that the sample size is calculated by the consistency of early and regular OGTT, so the power of studying the direct association between hyperglycaemia measured in the early OGTT and adverse clinical outcomes will be low. However, since the regular OGTT with IADPSG criteria represents glycaemia that is associated with pregnancy outcomes, the consistency of the early OGTT and regular OGTT may reflect a possible association between early glucose metabolism and pregnancy outcomes. Another potential limitation is that the representative nature of the sample may be affected by selection bias.

In summary, there is currently limited evidence on the most appropriate gestational age for the OGTT. The purpose of this study is to investigate the diagnostic value of an OGTT performed at 18-20 gestational weeks. Pregnant women with GDM may potentially benefit from the result of the study by earlier diagnosis and management of GDM.
Author affiliations

${ }^{1}$ Department of Obstetrics and Gynecology, The First Affiliated Hospital of Sun Yat-sen University, Guangzhou, PR China

${ }^{2}$ Department of Endocrinology, The Sixth Affiliated Hospital of Sun Yat-sen University, Guangzhou, PR China

${ }^{3}$ Department of Laboratory Medicine, The First Affiliated Hospital of Sun Yatsen University, Guangzhou, PR China

${ }^{4}$ Department of Medical Statistic and Epidemiology, School of Public Health, Sun Yat-sen University, Guangzhou, PR China

Acknowledgements The authors thank Professors Jianping Zhang from the Department of OBGYN, Sun Yat-sen Memorial Hospital, Sun Yat-sen University, Yanbin Li from the Department of Endocrinology, the First Affiliated Hospital of Sun Yat-sen University and Wen Xu from the Department of Endocrinology, the Third Affiliated Hospital of Sun Yat-sen University for a critical review of the study design.

Contributors $B L$ designed the study together with $Y X$ and drafted the manuscript. YZ, JC and JY contributed to refine the procedures of participation and follow-up. LD contributed to the design of the laboratory methods. YL developed the data collection database. JZ contributed to the statistical analysis and critically reviewed the manuscript. ZW input all authors and researchers and organised the study procedures.

Funding This study is funded by the Sun Yat-Sen University Clinical Research 5010 Program (grant number 2016014, to BL). The study is in part funded by the National Natural Science Foundation of China (grant number 81300493, to BL) and Ph.D. Programs Foundation of Ministry of Education of China (grant number 20130171120070, to BL).

Competing interests None declared.

Ethics approval The study was approved by the Ethical Committees of The First Affiliated Hospital of Sun Yat-sen University (number 2016-042).

Provenance and peer review Not commissioned; externally peer reviewed.

Open Access This is an Open Access article distributed in accordance with the Creative Commons Attribution Non Commercial (CC BY-NC 4.0) license, which permits others to distribute, remix, adapt, build upon this work noncommercially, and license their derivative works on different terms, provided the original work is properly cited and the use is non-commercial. See: http:// creativecommons.org/licenses/by-nc/4.0/

\section{REFERENCES}

1. National Institutes of Health consensus development conference statement: diagnosing gestational diabetes mellitus, March 4-6, 2013. Obstet Gynecol 2013;122(Pt 1):358-69.

2. Landon $M B$, Spong $C Y$, Thom E, et al. A multicenter, randomized trial of treatment for mild gestational diabetes. $N$ Engl J Med 2009;361:1339-48.

3. Diabetes in pregnancy: management of diabetes and its complications from preconception to the postnatal period. National Institute for Health and Care Excellence. London: Clinical Guidelines, 2015.

4. American Diabetes Association. Standards of medical care in diabetes-2015 abridged for primary care providers. Clin Diabetes 2015;33:97-111.

5. Gupta Y, Kalra B, Baruah MP, et al. Updated guidelines on screening for gestational diabetes. Int $J$ Womens Health 2015;7:539-50.

6. Metzger BE, Gabbe SG, Persson B, et al., International Association of Diabetes and Pregnancy Study Groups Consensus Panel. International Association of Diabetes and Pregnancy Study Groups recommendations on the diagnosis and classification of hyperglycemia in pregnancy. Diabetes Care 2010;33:676-82.

7. Committee on Practice Bulletins-Obstetrics. Practice Bulletin No. 137: Gestational diabetes mellitus. Obstet Gynecol 2013;122(Pt 1):406-16.

8. Clausen TD, Mathiesen ER, Hansen T, et al. Overweight and the metabolic syndrome in adult offspring of women with diet-treated gestational diabetes mellitus or type 1 diabetes. J Clin Endocrinol Metab 2009;94:2464-70.

9. Kim SY, England JL, Sharma JA, et al. Gestational diabetes mellitus and risk of childhood overweight and obesity in offspring: a systematic review. Exp Diabetes Res 2011;2011:541308. 
10. McMillen IC, Robinson JS. Developmental origins of the metabolic syndrome: prediction, plasticity, and programming. Physiol Rev 2005;85:571-633.

11. Clausen TD, Mathiesen ER, Hansen T, et al. High prevalence of type 2 diabetes and pre-diabetes in adult offspring of women with gestational diabetes mellitus or type 1 diabetes: the role of intrauterine hyperglycemia. Diabetes Care 2008;31:340-6.

12. Diagnostic Criteria and Classification of Hyperglycaemia First Detected in Pregnancy. WHO guidelines approved by the guidelines review committee. Geneva: World Health Organization, 2013.

13. Hod M, Kapur A, Sacks DA, et al. The International Federation of Gynecology and Obstetrics (FIGO) initiative on gestational diabetes mellitus: a pragmatic guide for diagnosis, management, and care. Int J Gynaecol Obstet 2015;131(Suppl 3):S173-211.

14. O'Sullivan JB, Mahan CM. Criteria for the oral glucose tolerance test in pregnancy. Diabetes 1964;13:278-85.

15. Classification and diagnosis of diabetes mellitus and other categories of glucose intolerance. National Diabetes Data Group. Diabetes 1979;28:1039-57.

16. Metzger BE. Summary and recommendations of the Third International Workshop-Conference on Gestational Diabetes Mellitus. Diabetes 1991;40(Suppl 2):197-201.

17. Virally M, Laloi-Michelin M. Methods for the screening and diagnosis of gestational diabetes mellitus between 24 and 28 weeks of pregnancy. Diabetes Metab 2010;36(Pt 2):549-65.

18. Brown CJ, Dawson A, Dodds R, et al. Report of the Pregnancy and Neonatal Care Group. Diabet Med 1996;13(Suppl 4):S43-53.

19. American Diabetes Association. Gestational diabetes mellitus. Diabetes Care 2000;23(Suppl 1):S77-9.

20. Metzger BE, Lowe LP, Dyer AR, et al., HAPO Study Cooperative Research Group. Hyperglycemia and Adverse Pregnancy Outcomes. N Engl J Med 2008;358:1991-2002.

21. American Diabetes Association. Diagnosis and classification of diabetes mellitus. Diabetes Care 2011;34(Suppl 1):S62-9.

22. Carpenter MW, Coustan DR. Criteria for screening tests for gestational diabetes. Am J Obstet Gynecol 1982;144:768-73.

23. Glueck CJ, Goldenberg N, Wang P, et al. Metformin during pregnancy reduces insulin, insulin resistance, insulin secretion, weight, testosterone and development of gestational diabetes: prospective longitudinal assessment of women with polycystic ovary syndrome from preconception throughout pregnancy. Hum Reprod 2004:19:510-21.

24. Moyer VA, U.S. Preventive Services Task Force. Screening for gestational diabetes mellitus: U.S. Preventive Services Task Force recommendation statement. Ann Intern Med 2014;160:414-20.

25. American Diabetes Association. Standards of medical care in diabetes-2016 abridged for primary care providers. Clin Diabetes 2016;34:3-21.

26. Kleinwechter H, Schäfer-Graf U, Bührer $\mathrm{C}$, et al., German Diabetes Association; German Association for Gynaecology and Obstetrics. Gestational diabetes mellitus (GDM) diagnosis, therapy and follow-up care: Practice Guideline of the German Diabetes Association (DDG) and the German Association for Gynaecology and Obstetrics (DGGG). Exp Clin Endocrinol Diabetes 2014;122:395-405.

27. Hajian-Tilaki K. Sample size estimation in diagnostic test studies of biomedical informatics. J Biomed Inform 2014;48:193-204.

28. Zhu WW, Yang HX, Wei YM, et al. Evaluation of the value of fasting plasma glucose in the first prenatal visit to diagnose gestational diabetes mellitus in China. Diabetes Care 2013;36:586-90.

29. Liu B, Chen $\mathrm{H}, \mathrm{Xu} \mathrm{Y}$, et al. Fetal growth is associated with maternal fasting plasma glucose at first prenatal visit. PLOS ONE 2014;9: e116352.

30. Liu B, Geng H, Yang J, et al. Early pregnancy fasting plasma glucose and lipid concentrations in pregnancy and association to offspring size: a retrospective cohort study. BMC Pregnancy Childbirth 2016;16:56

31. Catalano PM, Tyzbir ED, Roman NM, et al. Longitudinal changes in insulin release and insulin resistance in nonobese pregnant women. Am J Obstet Gynecol 1991;165(Pt 1):1667-72.

32. Bitó T, Nyári T, Kovács L, et al. Oral glucose tolerance testing at gestational weeks $<$ or $=16$ could predict or exclude subsequent gestational diabetes mellitus during the current pregnancy in high risk group. Eur J Obstet Gynecol Reprod Biol 2005;121:51-5. 